\title{
INTERDISCIPLINARIEDAD Y UNIVERSIDAD REFLEXIONES DESDE LA FACULTAD DE DERECHO DE LA UNAULA
}

\author{
Hernando Salcedo G utiérrez \\ Fernando Salazar Mejía \\ Hernando Roldán Salas \\ Grupo de Investigación Ratio Juris
}

Resumen: La búsqueda de la calidad es un imperativo para todas las instituciones que componen el sistema sociedad, pero para las instituciones de educación superior es, además, un compromiso ético-moral y político. Varias son las propuestas que indican cómo lograrla, y Colombia se la ha jugado con los "estándares mínimos de calidad" exigidos por el decreto 2566 de 2003. Entre otros elementos, el decreto deja entrever que sólo una educación interdisciplinaria podría asegurar la calidad. D esde la Universidad Autónoma hemos enfrentado ese reto y estamos dando pasos para que nuestros abogados no sean técnicos de las leyes sino analistas de las mismas y buscadores de respuestas a los múltiples problemas que enfrenta el país.

Palabras Clave: interdisciplinariedad, transdisciplinariedad, educación superior, Calidad, derecho, Unaula.

"L as unidades complejas, omo el ser humano 0 la sociedad, son multidimensionales; el ser humano es a la vez biológion,

psíquion, social, afectivo, racional. L a sociedad omporta dimensiones históricas, económicas, sociológicas, religiosas...

E l conocimiento pertinente debe reconocer esta multidimensionalidad e insertar allí sus informaciones: se podría no solamente aislar una parte del todo sino las partes unas de otras; la dimensión económica, por ejemplo, está en inter-retroacciones

permanentes con todas las otras dimensiones humanas;

es más, la economía conlleva en sí, de manera holográmica: necesidades, deseos, pasiones humanas, que sobrepasan los meros intereses económicos".

Edgar Morin 
A las puertas del siglo XXI, caracterizado por el proceso de globalización e internacionalización, los cambios suceden tan rápidos, los escenarios y variables a tener en cuenta para cualquier decisión son tantos, que, como hijos del siglo XX, nos resulta difícil a veces adaptarnos. Como ya lo dijo Borges, nos "tocaron, como a todos los hombres, malos tiempos para vivir".

Pero como todos los hombres de todas las épocas, estos son nuestros tiempos. Tiempos que, desde la concepción de Morin, no podemos comprender con la mirada clásica del siglo XX. Estos tiempos demandan de una formación inter y transdisciplinaria que permitan comprender lo uno en lo múltiple y lo múltiple en lo uno. Una mirada de red, que permita tejer y enlazar tanto como sea posible, conceptos y categorías y proponer teorías que permitan una comprensión tan amplia como el mundo que nos tocó vivir.

Construir instituciones educativas que no cercenen el conocimiento ni dividan en tan múltiples fragmentos la realidad, es el reto en estos nuevos tiempos. Construir instituciones que posibiliten interpretar el mundo social, objetivo y subjetivo sin recurrir a dogmatismos, que permitan comprender la diferencia antes que, como dice el poeta colombiano, sea "tarde para el hombre".

Sin lugar a dudas, es tarea de la universidad marcar el ritmo en esta transformación. Como institución clásica del saber, no puede ser menor a sus tiempos. Pero la Universidad somos todos. Directivos facilitando todo el andamiaje para que los académicos discutan, analicen y propongan teorías para interpretar el mundo, seduciendo de paso a los estudiantes por la variedad, elegancia y solidez de la argumentación. Propiciando así que se perpetúe la discusión vía argumentación y no vía armamentista.

Pensar una universidad que posibilite esta transformación, es hoy posible. La actual Constitución, los lineamientos sobre educación superior emanados en Colombia desde 1992 por la Ley 30, la Ley General de Educación, las recomendaciones hechas por la "comisión de los sabios" en Colombia al filo de la oportunidad en 1995, la Misión Nacional para la Modernización de la
Educación Superior, en 1996; el Plan D ecenal, el proceso de acreditación emprendido por muchas universidades, los estándares mínimos exigidos en el Decreto 2566 de 2003, son algunas de las reglamentaciones y orientaciones que podemos tomar.

Pero superior a los lineamientos legales, está nuestra realidad. La compleja situación por la que estamos atravesando en los últimos veinte años exige que el país sea pensado responsablemente.

La UNAULA es una institución universitaria que desde su Acta de Fundación se propuso ser una Universidad nueva y distinta, dedicada desde la ciencia y la libre cátedra a pensar el país. Encauzar todas estas posibilidades desde perspectivas epistemológicas integradoras, complejas, inter y transdisciplinarias, que permitan enfrentar con una mirada más amplia el contexto en que nos desenvolvemos, se convierta en el reto a superar en este primer decenio del siglo XXI.

\section{LA EDUCACIÓN SUPERIOR: RETOS PARA EL SIGLO XXI}

«Considerar la educación y la formación en relación con la cuestión del empleo no quiere decir que la educación y la formación se reduzcan a una oferta de cualificaciones.

La educación y la formación tienen por función esencial la integración social y el desarrollo personal, mediante la asunción de valores comunes, la transmisión de un patrimonio cultural y el aprendizaje de la autonomía»

Libro Blanco sobre Educación y Formación de la Unión Europea (CEE, 1995, pág. 4).

Para nadie es un secreto que hoy en el mundo se está conformando un nuevo orden económico y social. La lógica de la sociedad industrial de fines de siglo XX, conllevó a que cada vez más los países del mundo estén interrelacionados. Análisis provenientes de las más variadas corrientes de pensamiento, concuerdan en que, a corto plazo, y gracias al auge de las comunicaciones y al fácil acceso a la información a 
través de la Red Electrónica, todos los ámbitos de la actividad humana estarán aun más conectados, de modo que los gobiemos no podrán desentenderse de lo que ocurre en otras latitudes.

La estrategia de unirse en bloques comerciales y políticos que están llevando a cabo los países occidentales más desarrollados, ha posibilitado que puedan competir en el ámbito internacional con mayores ventajas. Así mismo, el alto desarrollo científico-técnico que han alcanzado le ha impuesto una lógica tal a la relación laboral, que cada vez más el conocimiento especializado se convierte en el capital de grandes masas de su población. La sociedad posindustrial ha traspasado ya el umbral de la llamada sociedad del conocimiento y avanza inexorablemente hacia la primacía de la inteligencia y del saber como principales factores de progreso social y económico. A modo de ilustración veamos los siguientes datos que trae López Rupérez : "A escala mundial la producción de software informátio -producto puro de la inteligencia humana- onstituye la principal fuente de valor añadido en el comercio internacional. E $n$ la elaboración de chips el costo de los materiales representa menos del $1 \%$ del costo total. Tanto en su producción como en la de todos aquellos sistemas que tienen en el chip su componente fundamental, la sustancia más preciosa es el saber asociado a su concepción y a su desarrollo. Recientemente, la A sociación de la Industria E lectrónica del Japón (E IA J) ha estimado que las nuevas actividades vinculadas a la sociedad de la información -tales como la telemática, el teletrabajo, la enseñanza a distancia, etc- podrían alcanzar en el último quinquenio del presente siglo la envergadura de las industrias electrónicas 0 del automóvil, lo que supone una cifra del orden de los 527.000 millones de dólares" ${ }^{1}$.

Con tal panorama, la educación y la formación con calidad se convierten en un objetivo fundamental de todos los países desarrollados. Pero, no es sólo el conocimiento específico, directamente vinculado al mundo del empleo, el que resulta seriamente afecta- do por la nueva dinámica mundial, sino que "el dominio de los conocimientos básicos, las formas de pensamiento avanzado y las competencias cognitivas de carácter general constituyen, en el momento presente, ingredientes indiscutibles de un capital humano de calidad y la mejor garantía de adaptación a exigencias de cualificación y a entornos profesionales francamente dinámicos" ${ }^{2}$.

La nueva visión del conocimiento y del saber, ahora sinónimos de progreso personal, económico y social alcanza, así mismo, a las familias y origina un aumento de sus expectativas con respecto al funcionamiento de las instituciones educativas, suponiendo que estas deben velar porque sus hijos alcancen los niveles profesionales adecuados para vivir y competir en esta nueva sociedad.

Por otro lado, el tercer mundo agudiza cada día más sus contradicciones internas. El alto índice de analfabetismo, de pobreza absoluta, de deterioro del ambiente natural y social, hace que grandes capas de su población intenten emigrar hacia países desarrollados que le brinden un mejor modo de vida, lo que a su vez ha hecho que estos países adopten políticas de migración más severas y restrictivas, aumentando la tensión entre países desarrollados y subdesarrollados.

Como nunca antes en la historia de la humanidad, hoy puede notarse la diferencia notoria entre desarrollo y subdesarrollo. La técnica y la ciencia, otrora pensadas para sacar a la humanidad de su minoría de edad, se han convertido también en fuente de dependencia y esclavitud para aquellos que no tienen acceso a ella. Por tanto, el reto para la humanidad del siglo XXI es cerrar la brecha entre aquellos que tienen la posibilidad de la información, la formación académica para darle un tratamiento diferente y producir con ella nuevo conocimiento, y aquellos que no tienen tales posibilidades. La educación juega aquí, por tanto, papel principal. En otras palabras, el reto

\footnotetext{
${ }^{1}$ López, Rupérez, Francisco. Hacia unos centros educativos de calidad. Contexto, fundamento y políticas de calidad en la gestión escolar. D ocumento de la Secretaría G eneral deE ducación y Formación Profesional del Ministerio de Educación y Cultura de España. Pág. 3.

${ }^{2}$ Ibíd. Pág. 4
} 
es construir un sistema educativo que permita la construcción, tal y como lo plantea Lipman, de pensamiento de alto orden.

Por todo lo anterior, se requiere de una profunda transformación del modelo educativo colombiano, y este proceso requiere de un gran compromiso de las universidades. La Universidad en occidente ha sido tradicionalmente cuna de la discusión racional. D esde ella se ha producido gran parte del bagaje teórico que nutre a la filosofía, el arte y la ciencia, cuestión que se debe a que en su interior se han conformado grupos de trabajo, análisis e investigación que han sido el soporte del imaginario colectivo universitario.

D esde esta perspectiva es tarea de la Universidad repensar los modelos educativos, los planes de estudio y los modelos de organización y gestión de nuestras instituciones educativas. Las nuevas propuestas deben propender por la flexibilización de los planes de estudio de modo tal que se permita a los alumnos la posibilidad de formarse también en otros espacios de la universidad, estableciendo así relaciones con otras disciplinas, espacios y momentos. Así mismo, apostarle a la construcción de estudiantes activos, creativos, pensadores de preguntas y respuestas propias de su entorno, formados para que desarrollen las competencias apropiadas para triunfar en el mundo que les tocó vivir.

Ello implica pensar un nuevo docente universitario. Es ya común que el estudiante desde su casa tenga acceso a través de modernas redes de comunicación, a seminarios y conferencias impartidos por los mejores profesores, así como a documentos y materiales gráficos, animados e interactivos, de muy alta calidad. Por tanto, el docente debe concebirse más como un tutor, dispuesto a formar en las más altas habilidades de pensamiento y acción en sus estudiantes, dejando a un lado la enseñanza rígidamente memorística, por lo que habrá de cambiar su forma clásica de evaluar el aprendizaje. Un docente que valora los aspectos formativos más que los meramente informativos.

Una universidad así estructurada, dedicada a investigar las problemáticas propias de nuestro entorno sociocultural, se va convirtiendo en el eje de la transformación social que tanto necesitamos. Por tanto, formar a estas nuevas generaciones desde una perspectiva distinta a la meramente profesionalizante implica fortalecer la investigación y apostarle a miradas complejas y multidisciplinarias. Concepciones de investigación que permitan la construcción de comunidad académica y deje de lado celos profesionales o partidistas que entorpecen el desarrollo de las instituciones.

\section{LA EDUCACIÓN SUPERIOR EN COLOMBIA: EN BUSCA DE LA CALIDAD}

Que la realidad que hemos construido nos desbordó, es una afirmación cada vez más justificada. La educación colombiana, incluida la universitaria, fruto del paradigma clásico de ciencia y pedagogía, no pudo cumplir con su cometido de ofrecer respuestas a las demandas propias de nuestro entorno sociocultural. Como quedó mostrado, nos encontramos en un escenario en donde la cotidianidad invita a ser leída y comprendida desde esquemas mentales amplios, frutos del trabajo interdisciplinario. Una mirada holística, compleja en contraposición con la clásica mirada cercenadora que nos vendió el paradigma positivista.

En esta medida, se vienen planteando desde la década de los sesenta cambios estructurales en la universidad colombiana que apuntan a mejorar dos puntos específicos: La calidad y la cobertura. El Ministerio de Educación viene impulsando actualmente reformas en pro de la calidad de los centros docentes. Su meta es mejorar los resultados de nuestro sistema educativo mediante el desarrollo de un conjunto de políticas centradas en la institución escolar pero, sobre todo, en los sujetos que la componen.

Pensar unas políticas de desarrollo centradas en los sujetos implica por supuesto pensar ya en la calidad. Es urgente que la universidad en Colombia, luego de años de centrar su existencia en el aumento cuantitativo, empiece a implementar políticas que recojan las tantas recomendaciones de directivas ministeriales y de la comunidad académica. Es urgente producir y 
aplicar conocimientos orientados a la generación de bienes y servicios; la necesidad de disponer de un talento humano altamente calificado. Ello implica, como bien lo apuntan las directrices del ICFES, "articular un conjunto de reglas de juego que recojan los <mínimos> acordados" ${ }^{\prime 3}$. Estos mínimos acordados que apuntan a la obtención de una educación superior con calidad y pertinente, se recogen en la propuesta de unos "ESTÁNDARES MÍNIMOSDE CALIDAD", según decreto 2566 de 2003.

Inscribirse hoy en una propuesta que busque "redimensionalizar las instituciones de educación superior, y posibilitar en ella desarrollos cualitativos en sus formas de organización académica, gestión y desarrollo del conocimiento", es comprometerse en la obtención de unos estándares mínimos de calidad 4 . Es un intento por renovar las viejas estructuras universitarias, rígidas y cerradas, centradas en epistemologías positivistas y reivindicadoras de lo disciplinar, por estructuras flexibles, integrales, complejas, centradas en epistemologías constructivistas y con propuestas cognitivas pertinentes.

Obtener unos estándares mínimos de calidad, tal y como lo plantea el decreto, ha implicado un desafío para nuestra universidad. Es necesario concebirlos más allá de un conjunto de requisitos legales para seguir funcionando un programa y aprovecharlos para replantear nuestros modelos pedagógicos y curriculares, nuestras prácticas educativas cotidianas y pensar en el desarrollo de las competencias que necesitan nuestros profesionales. Redefinir el marco conceptual que sobre la ciencia, la tecnología, la investigación y las prácticas disciplinares tenemos en la actualidad.

Lo mismo podríamos decir desde tal decreto del sistema de créditos.

Según Díaz, los estándares de calidad y el sistema de créditos se han asociado a la modernización de los programas en el país, modernización que debe surgir de, y ser consonante con, la reconceptualización y transformación de las profesiones a partir $\mathrm{de}^{5}$ :

a) El análisis del estado actual del conocimiento de las profesiones.

b) La (de)-(re)construcción de los programas de formación profesional tradicionalmente ordenados en asignaturas jerarquizadas, estratificadas y mediadas por secuencias rígidas y ritmos de formación inflexibles.

c) La reconceptualización de los principios de orden social que se requieren para afrontar de manera crítica los cambios de orden económico, cultural y tecnológico.

d) La introducción de formas de organización del conocimiento integradas y de modalidades de relación social participativas y colaborativas que activen nuevas competencias socio afectivas de profesores y estudiantes y favorezcan su desarrollo personal, social e intelectual, proclive al cambio.

e) El replanteamiento crítico de la formación profesional no sólo a partir de potencialidades del mercado educativo, sino también de las tendencias más modemas que inspiran el ejercicio profesional.

f) La reorganización de los arreglos internos básicos que soportan el desarrollo de las profesiones (físicos, académicos, tecnológicos, económicos, administrativos) en las instituciones de educación superior.

g) El replanteamiento de la excesiva profesionalización expresada en currículos sesgados por las especialidades de una profesión y aun por la especificidad de las ocupaciones.

h) El replanteamiento de la formación en el tiempo y espacio.

i) La redefinición de los propósitos deformación que articulen una gama de competencias, actitudes, valores, responsabilidades y compromisos en la formación.

En el mismo sentido, el sistema de créditos apunta a propiciar:

\footnotetext{
${ }^{3}$ ICFE S. Bases para una política de Estado en materia de educación superior. Bogotá, Marzo de 2001. Página 23.

${ }^{4}$ Diaz, Mario. Estándares mínimos de calidad. En “Elementos de política para la educacion superior”. ICFES, Bogotá, 2002.

${ }^{5}$ D íaz, Mario. Los estándares de calidad. En obra citada. Página 18-19.
} 
a) Mayor autonomía del estudiante para elegir actividades formativas que respondan a sus intereses y motivaciones.

b) El fomento de la producción y el acceso a diferentes tipos de experiencias de aprendizaje flexible.

c) Una clara organización de los deberes del estudiante en los periodos académicos.

d) Un ajuste del ritmo del proceso de formación a las diferencias individuales de los estudiantes.

e) La oferta de actividades académicas nuevas y variadas por parte de la institución.

f) Homologar con mayor facilidad las distintas asignaturas y programas.

g) Formarse en diferentes escenarios ${ }^{6}$.

\section{EL DISCURSO UNIVERSITARIO: DE LA RACIONALIDAD DISCIPLINAR A LA RACIONALIDAD INTER Y TRANSDISCIPLINAR.}

La forma más elaborada y eficaz que hemos empleado en O ccidente para enfrentar los problemas, ha sido la racionalidad instrumental, fruto de un tipo de reflexión sobre la ciencia. Desde ella y su particular forma de enfrentar los problemas, hemos dividido el mundo en múltiples partecitas para poder conocerlo concienzudamente. Así, la super-especialización en una disciplina ha sido la estrategia para lograr tal objetivo.

Ello ha hecho que las universidades (¿universitas?) propongan facultades que se encargan de ciertas parcelas de la realidad, las que a su vez proponen programas (pregrados) que se especializan aún más. Paradójica conclusión: la universitas sólo permite ver una pequeñísima parte del todo. Como dice Edgar Morin, lo que las universidades nos proporcionan es un «conocimiento ciego».

La división del saber, la exagerada especialización, la separación entre disciplinas, hoy son, más que ventajas, graves inconvenientes a la hora de comprender la complejidad del mundo social y natural en que nos tocó vivir. Esa separación de saberes se torna inoperante cuando se enfrenta a la realidad concreta que vivimos. Cuando se enfrentan los problemas básicos y reales de la vida, que exigen saber cómo producir suficiente alimento para la población, cómo asegurarle una buena salud, cómo garantizar su seguridad personal, cómo bajar el índice de inflación, cómo aumentar la tasa de empleo laboral o cómo ofrecerle una explicación del sentido del universo, se queda corta esta mirada.

Este tipo de racionalidad no nos sirve hoy para enfrentar los retos del siglo XXI. Los problemas que enfrenta hoy la humanidad, no son los mismos que enfrentamos en siglos anteriores. Una revisión somera de la prensa de cada país nos muestra en el acto una serie de fenómenos que, queramos o no, nos tocan a todos; son universales. Son fenómenos o problemas que se caracterizan por dos asuntos: primero, son enormes, es decir, están por fuera de las normas conocidas y por mucho que lo intentemos no los podemos reducir. Segundo, al analizarlos empezamos a notar que se interconectan con otra serie de problemas o asuntos, es decir, se muestran en toda su complejidad.

Abordarlos implica utilizar una estrategia distinta a la disciplinar. Una estrategia de pensamiento que en vez de reducir la complejidad de nuestras relaciones, las amplíe. Una estrategia de pensamiento que permita ver las múltiples y ricas relaciones que se dan entre el todo y la parte, y viceversa. Una estrategia de pensamiento inter y transdisciplinar.

Vistas así las cosas, "la interdisciplinariedad puede significar pura y simplemente que diferentes disciplinas se sientan en una misma mesa, en una misma asamblea, como las diferentes naciones se reúnen en la ONU sin poder hacer otra cosa que afirmar cada una sus propios derechos nacionales y sus propias soberanías en relación a las usurpaciones del vecino.

${ }^{6}$ Ibíd. Págs. 28-29 
Pero interdisciplinariedad puede también querer decir intercambio y cooperación, lo que hace que la interdisciplinariedad puede devenir en alguna cosa orgánica. La polidisciplinariedad constituye una asociación de disciplinas en virtud de un proyecto o de un objeto que le es común; mientras que las disciplinas son llamadas como técnicas especializadas para resolver tal o cual problema, en otros momentos, por el contrario, están en profunda interacción para tratar de concebir este objeto y este proyecto, como en el ejemplo de la hominización. En lo que concierne a la transdisciplinariedad, se trata a menudo de esquemas cognitivos que pueden atravesar las disciplinas, a veces con una virulencia tal que las coloca en dificultades. De hecho, son complejas cuestiones de ínter, de poli, y de transdisciplinariedad que han operado y han jugado un rol fecundo en la historia de las ciencias; se debe retener las nociones claras que están implicadas en ellas, es decir, la cooperación, y mejor, articulación, objeto común y mejor, proyecto común"7.

Tal como lo afirma González ${ }^{8}$ la Interdisciplinariedad, puede entenderse "como la transferencia de métodos de una disciplina a otra. Este intercambio, puede expresarse en distintos grados. A saber, de acuerdo al grado de aplicación, lo que se traduce en ocupar métodos de otras disciplinas: por ejemplo en el ámbito de las ciencias sociales, usar los métodos etnográficos en el estudio de procesos psicológicos. En un grado epistemológico, se tratará de usar fundamentos - una episteme determinada- de otras disciplinas para comprender y producir conocimiento. En el caso de la disciplina psicológica y los modos comprensivos usados para abordar la subjetividad, muchas veces refieren a fundamentos y elaboraciones producidas en el campo de la historia y la filosofía. Por último, un tercer grado de interdisciplinariedad, estaría dado por la concepción de nuevas disciplinas como efecto de lo "Inter", es decir, productivizar el cruce disciplinar para la fundación de una disciplina otra".
La interdisciplinariedad concierne a la transferencia de métodos de una disciplina a otra. Se pueden distinguir tres grados de interdisciplinariedad: a) un grado de aplicación. Por ejemplo, los métodos de la física nuclear transferidos a la medicina conducen a la aparición de nuevos tratamientos del cáncer; b) un grado epistemológico. Por ejemplo, la transferencia de los métodos de la lógica formal en el campo del D erecho genera análisis interesantes en la epistemología del D erecho; c) un grado de concepción de nuevas disciplinas. Por ejemplo, la transferencia de los métodos de la matemática en el campo de la física ha engendrado la físico-matemática, de la física de las partículas a la astrofísica -la cosmología cuántica, de la matemática a los fenómenos meteorológicos o los de la bolsa -la teoría del caos, de la informática en el arte- el arte informático. Como la pluridisciplinariedad, la interdisciplinariedad sobrepasa las disciplinas pero su finalidad queda inscrita en la investigación disciplinaria. Por su tercer grado, la interdisciplinariedad contribuye al gran «bang» disciplinario.

Siguiendo a Morin, podemos entender la Transdisciplinariedad como la respuesta del espíritu frente a la fragmentación y dispersión de los conocimientos que no pueden enfrentar la emergencia de los fenómenos complejos. Es un tipo de pensamiento que relaciona, une, teje. Trata de responder a las exigencias de nuestra actual realidad socio-cultural y universitaria. Se presenta como una estrategia de pensamiento que nos ayuda a poner en práctica un tipo de entendimiento en la que la articulación, la organización, sustituyen -no los eliminan- los modos analíticos de pensamiento. La transdisciplinariedad presupone las disciplinas pero las relativiza. Organiza en un saber de un nuevo tipo los diferentes saberes disciplinares en función de la multidimensionaldad de lo real. Promueve el diálogo abierto entre diversas perspectivas y diversas estrategias, para que nos aporten un conocimiento lo más integrador y contextualizador posible.

\footnotetext{
${ }^{7}$ Guarín Jurado, Germàn http:/ / www.ucm.edu.co/ final/ memorias_congreso/ Interdisciplinariedad.pdf.

${ }^{8} \mathrm{G}$ onzalez, Vivanco G abriela: D iscusión acerca de los límites de la Transdisciplinariedad. O de las posibilidades de un saber en tránsito y transferible. Universidad A cademia de Humanismo Cristiano. En línea.
} 
En la investigación Transdisciplinar, como dice Miguel Martínez , los participantes transcienden las propias disciplinas (o las ven sólo como complementarias) logrando crear un nuevo mapa cognitivo común sobre el problema en cuestión, es decir, llegan a compartir un maro epistémic amplio y una cierta meta-metodología que les sirven para integrar conceptualmente las diferentes orientaciones de sus análisis: postulados 0 principios básicos, perspectivas o enfoques, procesos metodológicos, instrumentos conceptuales, etc.

\section{INTERDISCIPLINARIEDAD Y TRANSDISCIPLINARIEDAD EN LA FACULTAD DE DERECHO DE LA UNAULA.}

La Facultad de Derecho de la Universidad Autónoma Latinoamericana, ha venido haciendo ingentes esfuerzos por ponerse a tono con los últimos aportes logrados en las ciencias sociales, tanto en lo relativo a los análisis sociales, políticos, económicos y culturales, como en lo relacionado con el estudio, la investigación y la producción de conocimiento en el área del D erecho y su relación con otras disciplinas afines, además de ajustarse a las exigencias de la ley de educación superior y de Colciencias.

En este sentido ha abordado un proceso que está relacionado básicamente con su consolidación y fortalecimiento del potencial académico construido durante 41 años de existencia y experiencia en la educación superior y que se ha expresado en la ocupación de lugares relevantes (puesto 15) entre las instituciones que ofrecen este programa a nivel nacional, y está ubicada en un NIVEL SUPERIOR.

En este proceso, se asume el cambio como la conciencia del contexto social, la época y las trasformaciones paradigmáticas ocasionadas en las formas de hacer, pensar, producir y relacionarse, en los nuevos centros del conocimiento, la producción, la organización de procesos y el surgimiento de otras formas de obligaciones, derechos y compromisos que pueden trascender los límites espaciales, territoriales e institucionales de los Estados nación y los mercados nacionales, generando otros escenarios de mayor complejidad de intercambio, socialización y sociabilidad. Para lo cual la Facultad estableció tres principios para enfrentar los procesos de innovación: Eticidad, Calidad y Responsabilidad; tres políticas institucionales: 1. transparencia, equilibrio y equidad, 2. Mejoramiento y evaluación continua y, 3 Compromiso con el respeto y el cumplimiento de los deberes y de las obligaciones: y tres estrategias a) Generación de información precisa, clara y veraz sobre el programa de Derecho, b) Diseño de estrategias y metodologías, además del fomento de actitudes, para la consecución de altos niveles de calidad académico administrativa, creación y seguimiento continuo de los procesos de autoevaluación y heteroevaluación y c) Acatamiento incondicional a la normatividad legal vigente. ${ }^{9}$ Así, se tiene la interdisciplinariedad como fundamento de las políticas y las estrategias institucionales para el cumplimiento de los objetivos de la malla curricular del programa de Derecho.

La interdisciplinariedad se expresa a través de todo el transcurrir del programa de la Facultad de Derecho de la UNAULA, específicamente en la construcción de la contextualización del programa: "los desafíos que el D erecho como disciplina y como referente simbólico de dominación debe resolver, en una época donde la interdisciplinariedad, la transdisciplinariedad y la multidisciplinariedad constituyen las nuevas estrategias paradigmáticas en la producción de conocimiento, en un contexto de crisis del monismo jurídico cuestionado por el pluralismo jurídico y el uso alternativo del Derecho y la judicialidad..." ${ }^{\prime 10}$

\footnotetext{
${ }^{9}$ Universidad Autónoma Latinoamericana, Facultad de D erecho. D ocumento para la obtención del registro calificado. Medellín, mayo 2007 pag. 4.

${ }^{10}$ Ibíd. pag. 9.
} 
En específico, la conciencia del momento y de la época se expresa en la constatación de la complejidad, integridad y espacialidad de los hechos, sujetos y relaciones sociales que han aportado las diferentes disciplinas científicas, como nuevas formas de explorar, pesquizar, analizar e interpretar las realidades objeto del conocimiento disciplinar y que básicamente se manifiestan en la articulación de la diversidad de lógicas, razonamientos y metodologías a través de la interdisciplinariedad, la ínterinstitucionalidad y la interaccionalidad (acción en red), para lo cual el proceso de organización curricular de la Facultad estableció las siguientes situaciones:

1. Se ubica la Facultad en un contexto de globalización y transformaciones internacionales, regionales, nacionales y locales, que muestran cómo los diferentes procesos sociales, económicos, políticos y culturales, se mueven en una trama de intercambio, interlocución y trascendencia mutua de los límites entre lo global y lo local ${ }^{11}$.

2. La interdependencia, diálogo e interacción entre la docencia, la investigación y la extensión social, como funciones básicas que desarrolla toda institución de educación superior, verificada en el documento entregado para el registro calificado en la parte de la justificación $^{12}$ relacionada con los cambios operados en el ejercicio de la profesión del Derecho, el impacto logrado y los desafíos a enfrentar.

3. La creación de un Centro de Investigaciones Sociojurídicas en cuyo diseño institucional y política de investigaciones se resalta la interdisciplinariedad no sólo en términos metodológicos sino en la conformación de los grupos de investigación: abogados, sociólogos, licenciados en ciencias sociales y educación, además del diseño de los proyectos de investigación con un abordaje pluridisciplinar de los objetos y problemáticas a investigar, que van desde cuestiones de la justicia y del derecho y las decisiones gubernamentales hasta la ciudadanía y los derechos fundamentales.
4. Además de ello, el modelo pedagógico es construido con base en la concepción de la teoría de la complejidad y el constructivismo, las cuales son aleccionadoras acerca de la inter y transdisciplinariedad y otras formas que adquieren las prácticas de la enseñanza-aprendizaje en sus modalidades educativas, investigativas y de intervención social o extensión. Y siendo coherentes con ello, hemos venido desarrollando las asignaturas tomando como base la solución de problemas. Nótese que el plan de estudios está estructurado de forma tal, que la investigación le es transversal: no sólo se trata de una asignatura denominada investigación en cada año, sino que cada curso está diseñado para que docentes y estudiantes aborden preguntas que surgen de las discusiones diarias de nuestro contexto social. Y ello sólo tiene sentido si luego se cruzan todos estos discursos. Ello lo logramos con las actividades de extensión, conferencias, artículos en nuestra revista y conversatorios.

5. La disposición articulada de las diferentes materias relacionadas con la producción, exploración, interpretación e investigación del conocimiento jurídico, como lógica y teoría del conocimiento, epistemología, filosofía y las metodologías de investigación en una línea continua facilita el acceso a las múltiples miradas que exige el abordaje de la compleja realidad del presente.

6. La organización de la estructura básica curricular de la carrera de D erecho en áreas, obedece no sólo a la afinidad temática, sino, también a la posibilidad de dialogar en términos de prácticas y discursos, didácticas y aprendizajes, metodologías y procedimientos, que faciliten un diálogo de conocimientos y saberes entre las diversas especialidades del discernimiento jurídico y social. En dicha organización hacen presencia métodos, juicios y saberes de disciplinas jurídicas, políticas, humanísticas y sociológicas. Por ello, los énfasis del programa en derechos humanos fundamentales y derecho público implican una visión inter y

${ }^{11}$ Ibíd. pag 9

${ }^{12}$ Ibíd pag 10 
transdisciplinar, en una realidad donde el reconocimiento de la multiculturalidad, la pluralidad y los derechos sociales de los grupos desaventajados, constituyen otra forma de dignificación de hombres y mujeres a partir de la diferencia, la distinción y la diversidad. ${ }^{13}$

7. Somos de los pocos programas que en la ciudad tienen en su plan de estudios un fuerte énfasis en asignaturas humano-sociales. Así, en el compara- tivo hecho en la justificación de nuestro programa con otros de la ciudad puede notarse que sociología jurídica, teoría del estado, derechos humanosD IH y solución alternativa de conflictos, son asignaturas que no tienen peso en los otros programas, y en la UNAULA son muy importantes. Pero, aunado a ello, en las asignaturas de énfasis proponemos una variedad de asignaturas que complementan los saberes específicos de las disciplinas, tal y como se muestra en el siguiente cuadro:

\section{LINEA DE ÉNFASIS EN DERECHO PÚBLICO}

\begin{tabular}{|l|l|l|}
\hline Derechos Fundamentales & Derecho Penal & Derecho Administrativo \\
\hline Asignaturas & Asignaturas & Seminarios \\
\hline Globalización y D erechos Humanos & Medicinal Legal & D erecho Disciplinario \\
\hline Medio Ambiente y D esarrollo & Criminología & Contratación Estatal \\
\hline $\begin{array}{l}\text { Derechos Fundamentales y } \\
\text { Jurisprudencia Constitucional } \\
\text { en Colombia. }\end{array}$ & $\begin{array}{l}\text { Derecho Probatorio Penal } \\
\text { y Criminalística }\end{array}$ & Derecho Laboral Administrativo \\
\hline Acciones Constitucionales & & Derecho Urbanístico \\
\hline Derechos Humanos Laborales & & Derecho Tributario \\
\hline
\end{tabular}

\section{LÍNEA DE ÉNFASIS EN DERECHO PRIVADO}

\begin{tabular}{|c|}
\hline Asignaturas \\
\hline Notariado y Registro \\
\hline Derecho de Seguros \\
\hline Daño Resarcible \\
\hline Propiedad Intelectual \\
\hline Teoría Económica \\
\hline
\end{tabular}

8. Una de las políticas del programa de Derecho de la Universidad Autónoma Latinoamericana lo constituye la internacionalización e interdisciplinariedad, como concreción del principio de la universalidad que se estableció para este programa ${ }^{14}$. Este principio es fundamento del programa que adquiere forma en la malla curricular.
9. La implementación del idioma extranjero, inglés, conecta el programa de nuestra Facultad de D erecho no sólo en las necesidades surgidas de los procesos de internacionalización que se vienen dando en el mundo sino, también, con esa multivariedad de esencias lingüísticas de carácter fonético, sintáctico, morfológico, ortográfico y semántico, enriquecedoras del conocimiento en

${ }^{13}$ Ibíd. Pág. 18 y 23.

${ }^{14}$ Ibíd. Pág. 22 
diversos ámbitos pero en, especial, en lo que podría denominarse la trilogía Derecho-LenguajeCosmovisión del mundo.

10.La introducción de la interdisciplinariedad, es un proceso que requiere acumulados, disposiciones y potencialidades, para lo cual, la Universidad y su Facultad de D erecho han desarrollado una serie de cambios institucionales como la inversión en docentes de alta calificación, doctorados y maestrías, las cuales representan un $50 \%$ del total del profesorado, y en capacitación de su capital humano en altos niveles de postgrado, para que este diálogo e intercambio de métodos, metodologías, juicios, enfoques epistemológicos se constituyan en fundamento de la educación, la investigación y la extensión social que desarrolla la Facultad de D erecho. El examen realizado hasta hoy, los cambios implementados y el proceso de autoevaluación realizado hasta el presente así lo han aconsejado.

11.Es característica de la disciplina del Derecho la interdisciplinariedad en la configuración de sus objetos, sujetos, espacios y sistemas que integran la producción del conocimiento jurídico y el ejercicio de la profesión en los distintos ámbitos sociales, específicamente la docencia y la investigación, propias del proceso de enseñanza aprendizaje del campo jurídico. El D erecho se fundamenta en los aportes epistémicos, métodos $\mathrm{y}$ metodologías, de las disciplinas humanas y sociales, como la filosofía, la lógica, la psicología, la sociología, la antropología, la economía, la política y otras artes y los oficios que enriquecen el conjunto de las civilizaciones, para enfrentar la magnitud de su esfera del conocimiento que estudia:1) las formas de regulación, ordenación y control de las relaciones sociales en los diferentes campos, ámbitos, espacios y escenarios, públicos 0 privados, de la acción social, 2) las obligaciones y derechos que surgen de los distintos intercambios, transferencias y compromisos, entre el Estado y los ciudadanos, las instituciones y el Estado, entre los particulares y entre las instituciones como tal, 3) la imperatividad normativa, las formas y principios racionales y materiales de validez y cumplimiento de la norma jurídica y su organización en sistemas jurídicos acordes con las culturas, las sociedades, su historia, mentalidades, imaginarios y referentes simbólicos de poder, control, orden, garantía, reconocimiento y respeto de los derechos, prestaciones y facultades de los ciudadanos.

En fin, la malla curricular de la Facultad de Derecho de la UNAULA parte de considerar la realidad como un complejo en el que convergen diferentes momentos, escalas, mesetas, contextos, en los cuales son leídos los objetos del conocimiento, en donde la interdisciplinariedad es considerada como una dinámica de reconocimiento y conocimiento de la realidad objeto de estudio, mediante la cual métodos y metodologías de disciplinas diversas, configuran el conjunto de estrategias de abordaje, enfoques interpretativos, nichos epistemológicos, que permiten ordenar lógicamente el proceso de producción de conocimiento.

Sintetizando, podemos afirmar que el programa de Derecho de la Universidad Autónoma Latinoamericana está impregnado de contenidos que responden interdisciplinariamente a las grandes inquietudes que hoy aquejan a la sociedad del siglo XXI. El mundo clama por la defensa de los D erechos Humanos Fundamentales; los seres humanos, y por ende la mayoría de los Estados de todo el orbe buscan afanosamente soluciones conjuntas a los gravísimos problemas que afectan el medio ambiente, entre los que se destacan los cambios climáticos y el deterioro de la capa de ozono; los comerciantes e industriales de todo el planeta buscan desaforadamente protecciones globales a la Propiedad Industrial, aún más allá de lo preceptuado, por ejemplo, en el Convenio de París, en el Acuerdo AD PIC o en las Decisiones de la Comunidad Andina de Naciones; También hoy se habla de un Derecho Urbanístico y comienza apenas a hacer carrera toda la teorización relativa a los denominados D erechos Humanos Fundamentales de índole Laboral. Y es en este contexto, aunado a las Teorías Económicas del sistema neoliberal contrastadas con otras cuyo perfil está orientado hacia una más adecuada y equitativa distribución de la riqueza, en el que el programa de la Facultad de D erecho se ubica hoy, y se perfila hacia el futuro, como un programa 
que está a la vanguardia de los nuevos saberes y de los nuevos requerimientos locales y globales manejados de manera interdisciplinaria y transdiciplinaria en la formación de lo que podríamos denominar los abogados del nuevo siglo.

Y es entonces, en el anterior marco globalizante, en el que el programa de la Facultad de D erecho se fortifica interdisciplinariamente haciendo énfasis con asignaturas tales como globalización y derechos fundamentales, medio ambiente y desarrollo, derechos fundamentales y jurisprudencia constitucional en colombia, derechos humanos laborales, derecho urbanístico, propiedad intelectual, teoría económica. Y es aquí, pero también en otros de los escenarios ya planteados, donde nuestro programa se conecta con otras disciplinas y con otros saberes que interactúan simultáneamente para producir, a la vez, una nueva cosmovisión del mundo y de la vida. Y esta cosmovisión del mundo y de la vida se traduce en preguntas que, desde el aula y más allá del aula, esquematizan problemas objeto de respuestas siempre provisionales (nunca definitivas), articulados a otras disciplinas incrustadas en el Plan de Estudios, y las cuales se ubican en los ámbitos de la LÓGICA, LA TEORÍA DEL CONOCIMIENTO, LA EPISTEMOLOGÍA, LA SOCIOLOGÍA, LA HERMENÉUTICA, y aún la MEDICINA LEGAL, como líneas conductoras de los procesos de investigación realizados a través de los semilleros y grupos de investigación.

La referida interdisciplinariedad de la malla curricular de la Facultad de Derecho también la encontramos en la inclusión de una segunda Lengua en el Plan de Estudios. En la "aldea global" del siglo XXI y, por ende, en los abogados de hoy y del mañana se percibe la imperiosa necesidad de mantener una comuni- cación fluida entre los seres de otras latitudes, y un mejor y mayor acercamiento hacia conocimientos expresados en idiomas diferentes al nuestro.

Somos conscientes que el trabajo inter y transdisciplinario es el presente de la producción del conocimiento en los centros de educación superior. Pero ello requiere de un largo camino que apenas en Colombia estamos empezando a recorrer. D esde nuestra Acta de Fundación estamos buscando ser una Universidad Nueva y D istinta, reconocimiento que en lo local nos hacen, pero que es un asunto arduo sobre todo en facultades de derecho donde siempre ha reinado la racionalidad instrumental. Sin embargo, tratamos de hacer eco de los artículos 11 y 14 de la carta de la transdisciplinariedad propuesta por Edgar Morin en 1994 en el seno del Primer Congreso de la Transdisciplinariedad, y que todos nuestros profesores y estudiantes conocen:

Artículo 11: Una educación auténtica no puede privilegiar la abstracción en el conocimiento. D ebe enseñar a contextualizar, concretar y globalizar. La educación transdisciplinaria reevalúa el rol de la intuición, del imaginario, de la sensibilidad y del cuerpo en la transmisión de los conocimientos.

Artículo 14: Rigor, apertura y tolerancia son las características fundamentales de la actitud y visión transdisciplinaria. El rigor en la argumentación, que toma en cuenta todas las cuestiones, es la mejor protección respecto de las desviaciones posibles. La apertura incluye la aceptación de lo desconocido, de lo inesperado y de lo imprevisible. La tolerancia es el reconocimiento del derecho a las ideas y verdades contrarias a las nuestras. 